\title{
Paeoniflorin inhibits nucleus pulposus cell apoptosis by regulating the expression of Bcl-2 family proteins and caspase-9 in a rabbit model of intervertebral disc degeneration
}

\author{
LIJUN SHI ${ }^{1}$, HONGLIN TENG ${ }^{2}$, MINYU ZHU ${ }^{2}, \mathrm{CHI} \mathrm{LI}^{2}$, KELUN HUANG ${ }^{2}$, \\ BI CHEN $^{2}$, YUSEN DAI ${ }^{2}$ and JING WANG ${ }^{2}$ \\ Departments of ${ }^{1}$ Pharmacy and ${ }^{2}$ Orthopedics, The First Affiliated Hospital of Wengzhou Medical University, \\ Wenzhou, Zhejiang 325000, P.R. China
}

Received August 1, 2014; Accepted April 13, 2015

DOI: 10.3892/etm.2015.2501

\begin{abstract}
Apoptosis plays a key role in the pathogenesis of internal disc disruption (IDD); therefore, the inhibition of apoptosis may offer a novel approach for treating IDD diseases. The aim of the present study was to investigate the effects and the underlying mechanisms of paeoniflorin through the detection of relevant indicators in a rabbit model of IDD. In total, 144 rabbits were used in the study and divided into four groups ( $\mathrm{n}=36$ per group). Rabbits successfully modeled with IDD received an intragastric injection of $120 \mathrm{mg} / \mathrm{kg} \cdot$ day paeoniflorin (high-dose group), $30 \mathrm{mg} / \mathrm{kg} \cdot$ day paeoniflorin (low-dose group) or saline (model saline group), while rabbits without IDD were used as a normal control group. The apoptosis rate of disc nucleus pulposus cells was detected using flow cytometry. In addition, the expression levels of Bcl-2, Bax and caspase-9 in the disc tissues were detected using immunohistochemistry and western blot analysis prior to and following the treatment. The results indicated that the expression levels of Bax in the low- and high-dose paeoniflorin groups were significantly reduced, while the Bcl-2 expression levels were significantly increased when compared with the model saline group $(\mathrm{P}<0.01)$. In addition, the expression levels of cleaved caspase-3 and cleaved caspase- 9 were reduced in the low- and high-dose paeoniflorin groups, as compared with the model saline group $(\mathrm{P}<0.05)$. Furthermore, the average apoptotic index of the high- and low-dose paeoniflorin groups was decreased when compared with the model saline group $(\mathrm{P}<0.05)$. In conclusion, paeoniflorin was demonstrated to inhibit the apoptosis of nucleus pulposus cells and the activation of caspase-3 and caspase- 9 through the regulation of Bcl-2 family protein
\end{abstract}

Correspondence to: Dr Jing Wang, Department of Orthopedics, The First Affiliated Hospital of Wengzhou Medical University, 2 Fuxue Road, Wenzhou, Zhejiang 325000, P.R. China

E-mail: wangjing7701@yeah.net

Key words: paeoniflorin, intervertebral disc degeneration, cell apoptosis, Bcl-2, Bax expression. These results provide an experimental basis for the future treatment of IDD with paeoniflorin.

\section{Introduction}

Internal disc disruption (IDD) involves the pathological alteration of intervertebral discs, which typically presents with virtually no evident morphological changes. Discogenic lower back pain is the primary manifestation of IDD. Heyde et al (1) observed that trauma is able to induce a caspase cascade as a response in affected disc cells, leading to the downregulation of the antiapoptotic protein, B-cell lymphoma 2 (Bcl-2), to subsequently result in cell apoptosis. Apoptosis plays a key role in IDD, and the inhibition of apoptosis may provide a novel treatment method for IDD diseases $(1,2)$. Therefore, inhibiting apoptosis in disc nucleus pulposus cells may be key for mitigating disc degeneration. Paeoniflorin is the primary active component isolated from the traditional Chinese medicinal herb Paeoniae Radix (3). Paeoniflorin exhibits a variety of types of biological activity, including anti-inflammatory (4), analgesic (4) and antioxidative (5) properties. Wu et al (6) suggested that plant-derived compounds such as paeoniflorin may possess the potential to prevent or treat cerebral ischemia and reperfusion-associated injuries.

In the present study, a rabbit IDD model was established, and intervention with the herbal extract, paeoniflorin, was conducted to investigate the effects of paeoniflorin on IDD and to determine the associated mechanisms through the detection of Bcl-2, Bax, caspase-3 and caspase-9.

\section{Materials and methods}

Experimental animals. In total, $144 \mathrm{New}$ Zealand white rabbits ( 82 male and 62 female; weight, $3.0 \pm 0.5 \mathrm{~kg}$ ) were provided by the Experimental Animal Center of the Academy of Medical Sciences [license no. SYXK(Zhe)2008-0114; Hangzhou, China]. This study was approved by the ethics committee of Wengzhou Medical University (Wenzhou, China).

Instruments. A paraffin section machine was obtained from Leica Biosystems GmbH (Nussloch, Germany). A BH-2 optical 
A Normal control Saline Low-dose high-dose

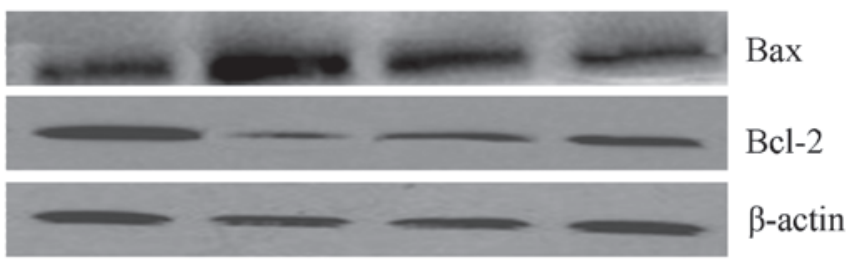

B

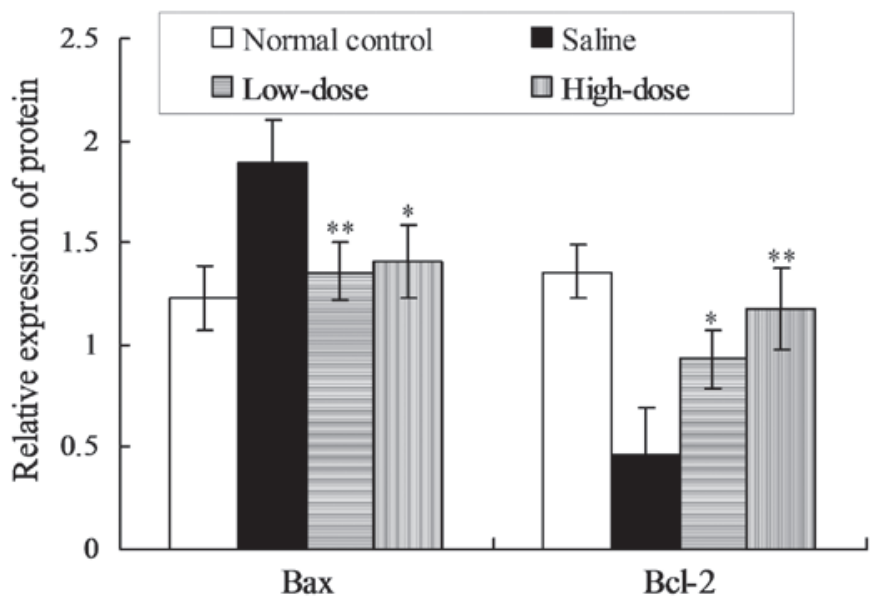

Figure 1. Effects of paeoniflorin treatment on Bcl-2 family protein expression. (A) Respresentative western blot showing the protein expression levels of Bcl-2 and Bax. (B) Statistical analysis of the Bcl-2 and Bax protein expression levels. " $\mathrm{P}<0.05$ and ${ }^{* *} \mathrm{P}<0.01$, vs. model saline group.

microscope and C5060 camera were obtained from Olympus Corporation (Tokyo, Japan). A ZHJH-1214 super clean bench was purchased from Suzhou Purification Engineering Installation Co., Ltd. (Suzhou, China). In addition, a TGL-16C centrifuge was obtained from the Shanghai Anting Scientific Instrument Factory (Shanghai, China), while a flow cytometer was purchased from Beckman Coulter (Brea, CA, USA).

Reagents and drugs. A streptavidin-biotin complex immunohistochemical staining kit was purchased from Fujian Maixin Biological Technology Ltd. (Fujian, China). Monoclonal antibodies targeting Bcl-2 (1:3,000; MAB4690) and Bax (1:4,000; H00000581-M01) were purchased from Abnova Corporation (Taipei, Taiwan), while a caspase- 9 polyclonal antibody $(1: 2,000)$ was obtained from BioVision, Inc. (3136-100; Milpitas, CA, USA). In addition, a monoclonal anti-Bax antibody $(1: 3,000)$ was purchased from BioLegend, Inc. (MMS-565R-100; San Diego, CA, USA), an anti-Bcl-2 antibody was purchased from Cell Signaling Technology, Inc. (Danvers, MA, USA) and a monoclonal anti-caspase-9 antibody $(1: 3,000)$ was purchased from LifeSpan BioSciences, Inc. (96-2-22; Seattle, WA, USA). Paeoniflorin was obtained from Ningbo Lihua Pharmaceutical Co., Ltd. (\#H20055058; Ningbo, China).

Modeling and grouping. In total, 144 New Zealand white rabbits were allocated at random into four groups $(n=36$ per group). An IDD model was established in the animals of the three experimental groups via an anular stab (7). All animals that subsequently exhibited IDD received an intragastric injection of $120 \mathrm{mg} / \mathrm{kg} \cdot$ day paeoniflorin (high-dose group), $30 \mathrm{mg} / \mathrm{kg} \cdot$ day paeoniflorin (low-dose group) or saline (model saline group), once per day. Animals that did not undergo the modeling procedure were used as a control group. Each animal was housed individually in a cage with free access to food and water.

Sample collection and indicator detection. Rabbits were sacrificed at weeks 3, 6 and 10 following surgery via an intraperitoneal injection of pentobarbital sodium $(1 \mathrm{ml} / \mathrm{kg})$, after which the intervertebral discs were separated and collected. Briefly, the nucleus pulposus was carefully removed and the gelatinous tissue was removed. The samples were fixed, paraffin-embedded and cut into 3-4 $\mu \mathrm{m}$ sections. Subsequently, the sections were affixed to precoated $10 \%$ poly-lysine slides (Sigma-Aldrich, St. Louis, MO, USA) and incubated at $63^{\circ} \mathrm{C}$ for $8 \mathrm{~h}$. The sections were stored at room temperature in preparation for staining, which was conducted using an Annexin V/propidium iodide double staining kit. The apoptosis rate of the disc nucleus pulposus cells was detected using flow cytometry.

Statistical analysis. All data were analyzed using SAS software, version 6.12 (SAS Institute, Inc., Cary, NC, USA). $\mathrm{P}<0.05$ was considered to indicate a statistically significant difference.

\section{Results}

Paeoniflorin inhibits the expression of Bax and promotes the expression of $\mathrm{Bcl}-2$. Protein expression levels of Bax and $\mathrm{Bcl}-2$ were detected using immunohistochemical and western blot analyses. The western blot assay results indicated that the expression levels of Bax in the low- and high-dose groups were 
Table I. Effects of paeoniflorin on the expression of Bax protein (\%).

\begin{tabular}{lccc}
\hline & & Positive cells & Week 10 \\
\cline { 2 - 4 } Group & Week 3 & Week 6 & $31.088 \pm 2.709$ \\
\hline Normal control & $28.574 \pm 2.375$ & $29.645 \pm 1.570$ & $50.343 \pm 1.404$ \\
Saline & $39.925 \pm 2.905$ & $46.023 \pm 3.570$ & $37.515 \pm 2.276$ \\
Low-dose & $33.693 \pm 2.496$ & $35.753 \pm 2.583$ & $32.800 \pm 1.562$ \\
High-dose & $31.645 \pm 1.280$ & $31.710 \pm 1.248$ & \\
\hline
\end{tabular}

Table II. Effects of paeoniflorin on the expression of Bcl-2 protein (\%).

\begin{tabular}{lccc}
\hline & \multicolumn{3}{c}{ Positive cells } \\
\cline { 2 - 4 } Group & Week 3 & Week 6 & Week 10 \\
\hline Normal control & $50.408 \pm 1.885$ & $50.455 \pm 1.830$ & $49.813 \pm 1.238$ \\
Saline & $38.695 \pm 1.285$ & $38.415 \pm 1.539$ & $36.503 \pm 2.525$ \\
Low-dose & $40.393 \pm 0.462$ & $40.620 \pm 0.526$ & $40.015 \pm 0.962$ \\
High-dose & $39.665 \pm 1.192$ & $38.813 \pm 1.242$ & $39.188 \pm 1.082$ \\
\hline
\end{tabular}

A

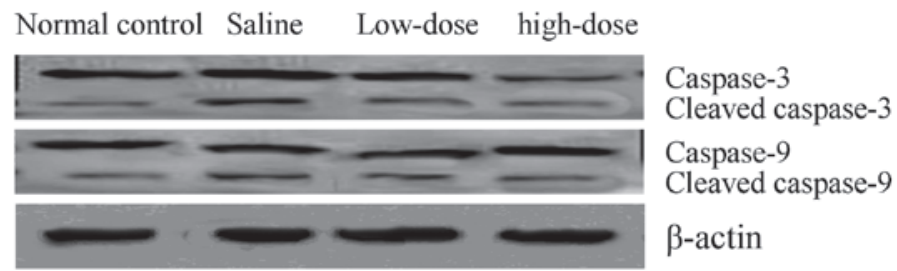

B

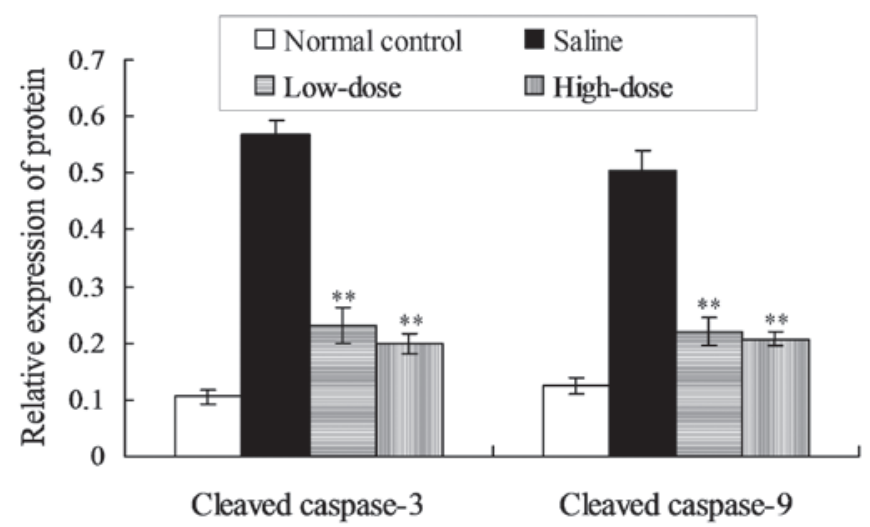

Figure 2. Effects of paeoniflorin treatment on the cleavage of caspase-3 and caspase-9 proteins. (A) Representative western blot showing the protein expression levels of cleaved caspase- 3 and caspase-9. (B) Statistical analysis of the cleaved caspase- 3 and caspase- 9 protein expression levels. ${ }^{* *} \mathrm{P}<0.01$, vs. model saline group.

significantly reduced when compared with the model saline group ( $\mathrm{P}<0.01$; Fig. 1). However, the expression levels of Bcl-2 in the low- and high-dose groups were significantly increased when compared with the model saline group $(\mathrm{P}<0.05$ and $\mathrm{P}<0.01$, respectively; Fig. 1).

Accordingly, the immunohistochemical analysis results demonstrated that the expression levels of Bax in the model saline group were elevated compared with the normal control group at weeks 3, 6 and 10 following surgery $(\mathrm{P}<0.05$; Table I). In addition, the expression levels of Bax in the low- and high-dose groups were reduced when compared with the model saline group $(\mathrm{P}<0.05$; Table I). No statistically significant difference was observed in Bax expression between the low- and high-dose groups at week 3 (P>0.05; Table I), while the expression level of Bax in the low-dose group was higher compared with the high-dose group at weeks 6 and $10(\mathrm{P}<0.05)$. 
Table III. Effects of paeoniflorin on the expression of caspase-9 protein (\%).

\begin{tabular}{lccc}
\hline & & Positive cells & \\
\cline { 2 - 4 } Group & Week 3 & Week 6 & Week 10 \\
\hline Normal control & $20.303 \pm 1.191$ & $20.853 \pm 1.402$ & $21.053 \pm 1.160$ \\
Saline & $28.355 \pm 0.897$ & $30.655 \pm 1.836$ & $31.043 \pm 2.07$ \\
Low-dose & $27.683 \pm 0.953$ & $28.233 \pm 0.819$ & $28.508 \pm 0.728$ \\
High-dose & $28.698 \pm 1.044$ & $28.923 \pm 1.270$ & $28.910 \pm 1.503$ \\
\hline
\end{tabular}

Table IV. Average apoptotic index (\%).

\begin{tabular}{lccc}
\hline & & Positive cells & Week 10 \\
\cline { 2 - 4 } Group & Week 3 & Week 6 & $4.90 \pm 2.86$ \\
\hline Normal control & $5.04 \pm 2.58$ & $5.10 \pm 2.42$ & $25.04 \pm 12.07$ \\
Saline & $29.35 \pm 11.86$ & $32.24 \pm 15.83$ & $20.89 \pm 11.72$ \\
Low-dose & $17.68 \pm 9.95$ & $20.23 \pm 11.82$ & $19.50 \pm 11.50$ \\
High-dose & $18.98 \pm 9.04$ & $19.92 \pm 12.27$ & \\
\hline
\end{tabular}

A

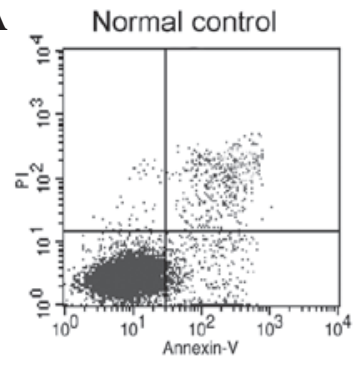

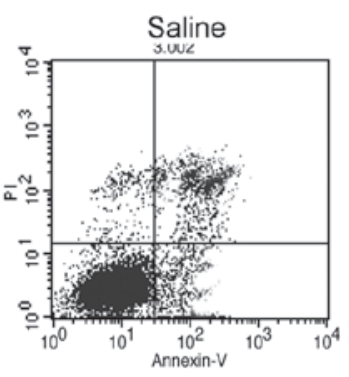
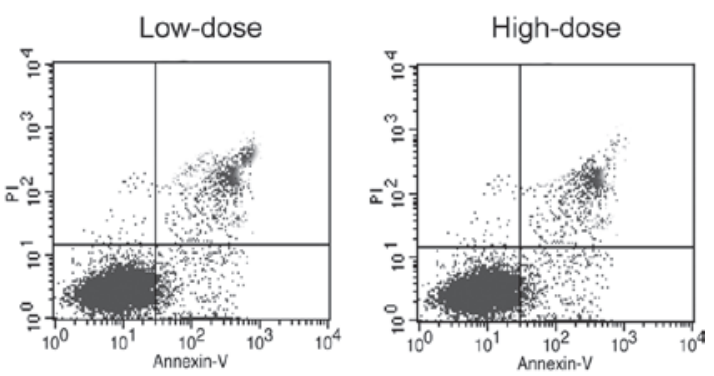

B

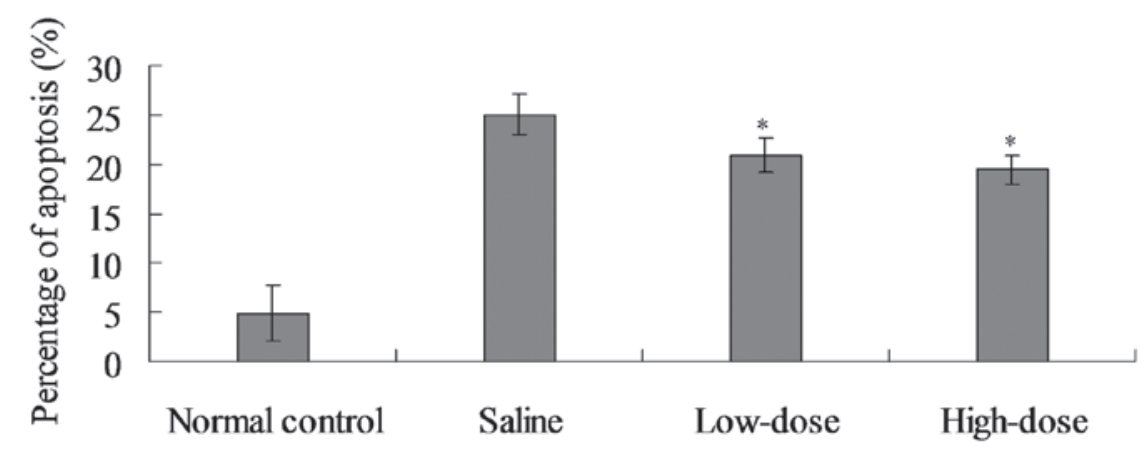

Figure 3. Detection of the apoptosis rate using flow cytometry. (A) Annexin V/PI double staining assays of the cells treated with or without paeoniflorin. The $\mathrm{x}$-axis indicates the number of PI-stained cells and the y-axis indicates the number of Annexin V-fluorescein isothiocyanate-stained cells. The percentage of cells labeled with only Annexin V (counted in the Q1 region) in each preparation are presented in each figure. Results for three independent experiments are shown. (B) Statistical analysis of the cell apoptosis rates. ${ }^{*} \mathrm{P}<0.05$, vs. model saline group. PI, propidium iodide.

Furthermore, the expression levels of Bcl-2 in the model saline group were reduced compared with the normal control group at weeks 3, 6 and 10 following surgery $(\mathrm{P}<0.05$; Table II). The expression levels of Bcl-2 in the high- and low-dose groups were reduced when compared with the model saline group $(\mathrm{P}<0.05$; Table II); however, no statistically significant differences were observed in the Bcl-2 expression levels between the high- and low-dose groups at weeks 3, 6 and 10 following surgery ( $\mathrm{P}>0.05$; Table II).
Paeoniflorin inhibits the activation of caspase-3 and caspase-9. Western blot analysis results indicated that the protein expression levels of cleaved caspase-3 and cleaved caspase-9 were reduced in the low- and high-dose paeoniflorin groups when compared with the model saline group $(\mathrm{P}<0.05$; Fig. 2).

Immunohistochemical analysis indicated that the expression of activated caspase- 9 in the model saline group was higher compared with the normal control group at weeks 3, 
6 and 10 following surgery $(\mathrm{P}<0.05$; Table III). At week 3, no statistically significant difference was detected in the activated caspase-9 expression when comparing the high- and low-dose groups with the saline group ( $\mathrm{P}>0.05$; Table III). However, at weeks 6 and 10, the activated caspase- 9 positive cell rates in the high- and low-dose groups were reduced, as compared with the saline group $(\mathrm{P}<0.05$; Table III). No statistically significant difference was observed in the activated caspase- 9 expression between the high- and low-dose groups $(\mathrm{P}>0.05$; Table III). Furthermore, the caspase- 3 exhibited comparable results to caspase-9.

Paeoniflorin reduces the disc cell apoptosis rate. The average apoptotic index of the model saline group was elevated compared with the control group at weeks 3, 6 and 10 following surgery $(\mathrm{P}<0.05$; Fig. 3 and Table IV). By contrast, the average apoptotic index in the high- and low-dose groups was reduced when compared with the model saline group $(\mathrm{P}<0.05$; Table IV). No statistically significant difference was detected in the average apoptotic index between the high- and low-dose groups (Table IV).

\section{Discussion}

Internal disc disruption (IDD) involves the pathological alteration of the intervertebral disc, while the overall intervertebral disc morphology exhibits no or limited modification. IDD can result in discogenic lower back pain, which is the primary manifestation of disc degeneration. Crock (8) was the first to hypothesize that discogenic pain may be caused by lesions of the disc internal structure. Furthermore, Heyde et al (1) observed that trauma was able to induce a caspase cascade response in affected disc cells and downregulate the expression of the antiapoptotic protein, Bcl-2, which subsequently resulted in cell apoptosis; a potential mechanism underlying the development of IDD.

Park et al (9) detected the expression levels of caspase-8, caspase-9, Bid and cytochrome $c$ in the degenerative disc nucleus pulposus cells of 32 patients with lumbar disc herniation. The authors observed that the apoptotic pathway of degenerative disc nucleus pulposus cells was predominantly mediated by the mitochondrial pathway. There are a limited number of death-inducing signaling complexes on the mitochondrial membrane, including caspase-8. Bcl-2 family members with a Bcl-2 homology $(\mathrm{BH}) 3$ domain are activated following stimulation by death signals, after which the proteins activate other Bcl-2 family members, such as Bax and Bak, to induce mitochondrial permeability and release cytochrome $c$ and other proteins $(10,11)$, thereby activating caspase- 9 and caspase- 3 to induce apoptosis (12). Bcl-2 family proteins can be divided into pro- and antiapoptotic proteins. Proapoptotic proteins can be further divided into multi-domain proteins, such as Bax and Bak, and BH3-only proteins, which include Bid, Bad, Bim, Bik, Bmf, Hrk, PUMA and Noxa. Antiapoptotic proteins include Bcl-2, Bcl-xl, Mcl-1 and Bcl-w.

The mechanism through which Bcl-2 inhibits apoptosis is considered to involve the inhibition of caspase proteins $(13,14)$. By contrast, Bax promotes caspase activity. $\mathrm{Bcl}-2$ is a key antiapoptotic gene, while Bax is representative of proapoptotic genes. Thus, the ratio between Bcl-2 and Bax may be used to estimate the cell sensitivity to apoptosis (15). The expression of Bax has been reported to increase, while the expression of Bcl-2 is decreased in degenerative lumbar disc tissues, indicating that $\mathrm{Bcl}-2$ and Bax are involved in the process of nucleus pulposus cell apoptosis, which destroys the dynamic equilibrium between cell proliferation and apoptosis, leading to the progression of lumbar disc degeneration $(16,17)$.

In conclusion, the present study used paeoniflorin to treat IDD, and the results indicated that the expression levels of Bax and caspase- 9 in the paeoniflorin groups were significantly reduced compared with the control group, while the expression of Bcl-2 was significantly increased. Through detecting the rate of apoptosis, the average apoptotic index values of the modeling groups were observed to be elevated compared with the normal control group $(\mathrm{P}<0.05)$. Furthermore, the average apoptotic index of the high- and low-dose paeoniflorin groups was reduced when compared with the model saline group $(\mathrm{P}<0.05)$. Therefore, the results of the present study indicate that paeoniflorin is able to regulate $\mathrm{Bcl}-2$ family proteins; promoting the expression of $\mathrm{Bcl}-2$ and suppressing the expression of Bax and caspase-9, to ultimately inhibit the apoptosis of nucleus pulposus cells. Therefore, the results of the present study provide an experimental basis for the treatment of IDD using paeoniflorin.

\section{Acknowledgements}

This study was supported by grants from the National Natural Science Foundation of China (no. 81202711), the Traditional Chinese Medicine of Zhejiang Province Science and Technology plan project (no. 2010ZA083) and the Natural Science Foundation of Zhejiang Province (no. LY12H06004).

\section{References}

1. Heyde CE, Tschoeke SK, Hellmuth M, Hostmann A, Ertel W and Oberholzer A: Trauma induces apoptosis in human thoracolumbar intervertebral discs. BMC Clin Pathol 6: 5, 2006.

2. Ye ZL, Hou XX, Chen RL, Ding J, Zheng GH, Chen MZ and Tian C: Effects of methylthiouracil on the proliferation and apoptosis of rat bone marrow stromal cells. Exp Ther Med 7: 1738-1744, 2014.

3. Kim ID and Ha BJ: Paeoniflorin protects RAW 264.7 macrophages from LPS-induced cytotoxicity and genotoxicity. Toxicol In Vitro 23: 1014-1019, 2009.

4. Zhang XJ, Li Z, Leung WM, Liu L, Xu HX and Bian ZX: The analgesic effect of paeoniflorin on neonatal maternal separation-induced visceral hyperalgesia in rats. J Pain 9: 497-505, 2008.

5. Chen T, Guo ZP, Jiao XY, Zhang YH, Li YH, Li JY and Liu HJ: Protective effects of peoniflorin against hydrogen peroxide-induced oxidative stress in human in human umbilical vein endothelial cells. Can J Physiol Phaarmacol 89: 445-453, 2011.

6. Wu PF, Zhang Z, Wang F and Chen JG: Natural compounds from traditional medicinal herbs in the treatment of cerebral ischemia/reperfusion injury. Acta Pharmacol Sin 31: 1523-1531, 2010.

7. Wang J, Tang TS, YangHL, Yao XS, Chen L, Liu W, and Li T: The expression of Fas ligand on normal and stabbed disc cells in a rabbit model of intervertebral disc degeneration: A possible pathogenesis. J Neurosurg Spine 6: 425-430, 2007.

8. Crock HV: Internal disc disruption. A challenge to disc prolapse fifty years on. Spine (Phila Pa 1976) 11: 650-653, 1986.

9. Park JB, Lee JK, Park SJ, Kim KW and Riew KD: Mitochondrial involvement in fas-mediated apoptosis of human lumbar disc cells. J Bone Joint Surg Am 87: 1338-1342, 2005. 
10. Eskes R, Desagher S, Antonsson B and Martinou JC: Bid induces the oligomerization and insertion of Bax into the outer mitochondrial membrane. Mol Cell Biol 20: 929-935, 2000.

11. Li J, Wang H, Ma Z, Fan W, Li Y, Han B, Zhang Z and Wang J: TAT-apoptin induces apoptosis in the human bladder cancer EJ cell line and regulates $\mathrm{Bax}, \mathrm{Bcl}-2$, caspase-3 and survivin expression. Exp Ther Med 3: 1033-1038, 2012.

12. Cain K, Bratton SB, Langlais C, Wakker G, Brown DG, Sun XM and Cohen GM: Apaf-1 oligomerizes into biologically active approximately $700-\mathrm{kDa}$ and in active approximately 1.4-MDa apoptosome complexes. J Biol Chem 275: 6067-6070, 2000.

13. Yang J, Liu X, Bhalla K, Kim CN, Ibrado AM, Cai J, Peng TI, Jones DP and Wang X: Prevention of apoptosis by Bc1-2: Release of cytochrome $c$ from mitochondria blocked. Science 275: 1129-1132, 1997.
14. Jung SY, Kim DY, Yune TY, Shin DH, Baek SB and Kim CJ: Treadmill exercise reduces spinal cord injury-induced apoptosis by activating the PI3K/Akt pathway in rats. Exp Ther Med 7: 587-593, 2014.

15. Gruber HE and Hanleyen N: Analysis of aging and degeneration of the human intervertebral disc-comparison of surgical specimens with normal controls. Spine (Phila Pa 1976) 23: 751-757, 1998.

16. Wang D, Liu M, Song H, Wang M, Yang K and Zhang Y: Expression of Bax and caspase-3 and apoptosis in human lumbar intervertebral disc degeneration. Zhongguo Xiu Fu Chong Jian Wai Ke Za Zhi 22: 421-425, 2008 (In Chinese).

17. Wang SL, Yu YL, Tang CL and Lv FZ: Effects of TGF-beta 1 and IL-1-beta on expression of ADAMTS enzymes and TIMP-3 in human intervertebral disc degeneration. Exp Ther Med 6: 1522-1526, 2013 